\title{
Design of Chain Drive Elevator in Complex Environment
}

\author{
Shuguang Niu, Xiaomei Jiang, Haizhou Zhang \\ School of Mechanical Engineering \\ Changshu Institute of Technology \\ Changshu, China \\ e-mail: niushuguang2000@163.com
}

\author{
Baoliang Meng \\ Nanjing Jiangning Market Supervision Administration \\ Jiangning district, Nanjing \\ Nanjing, China \\ e-mail: jszjjxm@hotmail.com
}

\begin{abstract}
This paper discussed the elevator towed forcibly by circular ring chain drive in complex environment, analyzed the key technology of the elevator design, and better solved the safety, maintenance and other problems of traction elevator by using chain drive with the advantages of maintenance free, corrosion resistance, without slip, high temperature resistance, cold resistance, withstanding the special weather and other adverse factors and so on.
\end{abstract}

Keywords—elevator; harsh environment; chain drive; reliability

\section{INTRODUCTION}

In 1903, Otis Elevator Company transformed from drum driven elevator to traction drive and built the foundations for the modern long stroke elevator. Wire rope of traction drive system is an important load component in the elevator which carries all the mass of the elevator car. In recent years, relative slip occurred between traction sheave and wire rope and the traction steel wire rope broke many times. The situation of elevator wire rope which is good or bad is directly relates to the safety of equipment and passengers. In general, sudden breaking of the elevator rope is seldom to be seen under normal conditions, which is caused generally by wear, broken wires, bending fatigue, corrosion or trauma in the long run[1]. Some designs aimed to improve the traction condition, adding a lower incision on the rope sheave groove, or adopting the $\mathrm{V}$ shaped groove design and even exceeded the wire rope capacity. Moreover, some designs took the use of reverse winding of rope or added a reverse guide pulley with a larger pressure but dramatically shortened service life of steel wire rope[2]. Moreover, these designs also increased the chance of wire rope side wear and the local stress concentration of the wire rope, which accelerated the damage of steel wire rope and even failure.

Lubrication of traction rope has great influence on the service life of the wire rope. If the lubrication is not reasonable, it will affect the elevator driven through friction and cause operation safety problems such as slip and etc.

\section{STRUCTURE DESIGN OF CHAIN DRIVE ELEVATOR}

Traction rope for suspension of the car and counterweight is substituted for circular ring chain, with sprocket driven circular ring chain replacing the traction pulley by friction transmission, following the drive sprocket forward and inversion without slip, thereby ensuring up and down of the car and counterweight along the guide rail. The elevator structure of circular ring chain driven by sprocket is shown in Fig.1.

\section{A. Design of circular ring chain and sprocket}

As a traction component of high power elevator, the tensile strength of circular ring chain is directly related to the reliability of the whole machine[3]. The tensile strength of circular ring chain is $3550 \mathrm{kN}$. With the increasing of the size of the circular ring chain, the requirement for the circular ring

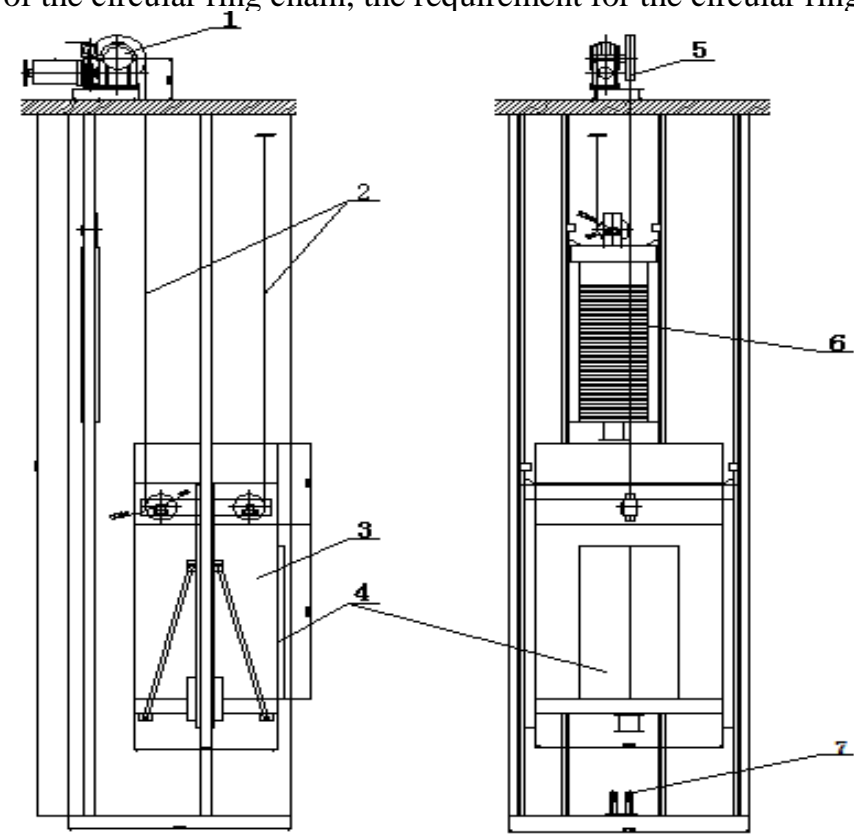

Fig.1 elevator structure of circular ring chain driven by sprocket. 1-traction motor, 2-circular ring chain, 3-car, 4-door, 5-drive sprocket, 6- counterweight 7- buffer 
chain is to improve the tensile strength and reduce the height of the vertical ring.

\section{B. Material and process}

(2)In order to improve the tensile strength of circular ring chain, 23MnNiCrM0542, 3MnNiCrM052 and $23 \mathrm{MnNicrM064}$ refining steel are widely used as the material of the large-sized ring chain at home and abroad. Welding process: the use of advanced welding equipment, using flash butt welding and annealing process, successfully solves the generation of overheat, over burn and other issues in the welding process. In terms of quality monitoring, metallography of the microstructure after welding and heat treatment of raw materials and tensile fracture by scanning electron microscope are analyzed; the load capacity and fatigue strength are greatly improved by using the mesh loop pre-stretching and strengthening technology. However, too high strength will reduce the toughness. If the chain is stuck in the course of operation and subjected to high impact load, it is especially easy to break[4]. We do an experiment on the steel sample composed of a high proportion of stable residual austenite after annealing. The results show that a high proportion of stable residual austenite steel effectively solves the above problems, the tensile strength is greater than 1600 $\mathrm{MPa}$ and toughness also satisfy the requirements.

$$
\begin{gathered}
\text { Ring chain pitch: } p=25.4 \mathrm{~mm} \\
\text { Breaking force: } \rho_{\max }=55.6 \mathrm{kN} \\
\text { Safety factor: } \Omega=55.6 / 3.5=15.9>8 \text {, safe }
\end{gathered}
$$

\section{Design of sprocket}

The sprocket assembly is the key element of the high power elevator after using the circular ring chain, its development trend is to improve the sprocket component reliability, enhance the machining precision of the sprocket, improve heat treatment process and prolong the service life of the sprocket. Currently, the sprocket assembly mostly adopts double row tapered roller bearing lubricated by dilute oil in the structure, active tail sprocket assemblies are the same as passive ones, the sprocket wheel adopts high - strength alloy steel, which is hardened and tooth surface hardness can reach HRc55 and effective hardening layer depth be more than 10 $\mathrm{mm}[5]$. In addition, the tooth shape of the sprocket is improved, the GR type sprocket that is designed has always three teeth contacted with chain links to drive the load instead of only one tooth to drive the entire load usually. Three teeth contact is bound to reduce the wear of sprocket and chain, thereby prolonging the service life of the sprocket and chain, reducing the elongation of the chain, and improve greatly the power transmission efficiency. Furthermore, chain achieves the best state in the life cycle, improves the safety and avoids an unforeseen accident.

Determination of the number of sprocket teeth: $\square \square$

- rough calculation of chain speed

$$
v_{\text {v }}=\frac{10 \times 1.25}{60}=0.21 \mathrm{~m} / \mathrm{s}
$$

- $\quad$ The small sprocket teeth number is 17 ;

$$
d=\frac{p}{\sin \frac{180^{\circ}}{17}}=\frac{25.4}{\sin \frac{180^{\circ}}{17}}=138.232 \mathrm{~mm}
$$

- Addendum circle diameter

$d_{n}=p\left(0.54+\operatorname{ctg} \frac{180^{\circ}}{z_{1}}\right)=25.4 \times\left(0.54+\operatorname{ctg} \frac{180^{\circ}}{17}\right)=149.594 \mathrm{~mm}$

- dedendum diameter

$d_{s}=d-d_{y}=138.232-15.88=122.352 \mathrm{mw}$

- $\quad$ pitch circle chordal height

$$
h_{n}=0.27 p=0.27 \times 25.4=6.858 \mathrm{~mm}
$$

- hub thickness

$h=R+\frac{d_{x}}{6}+0.01 d=6.4+\frac{19}{6}+0.01 \times 138.232=10.947 \mathrm{~mm}$

where: $\mathrm{K}$ - constant, $100<\mathrm{d}<150, \mathrm{~K}=6.4$

$d_{k}$. wheel hole diameter, $\mathrm{mm}$ Select $h=11 \mathrm{~mm}$

- tooth width

$b_{s}=0.95 b_{3}=0.95 \times 15.75=14.96 \mathrm{~mm}$

- tooth width

$$
b_{s 1}=0.96 b_{1}=15.12 \mathrm{~mm}
$$

\section{FINITE ELEMENT ANALYSIS OF CHAIN TRANSMISSION}

The comparison design and analysis are carried out by using circular ring chain in design, in accordance with the "GB / T 12718 - 2001 mining high strength ring chain" , the 18X64C high strength ring chain model is established in $\mathrm{PRO} / \mathrm{E}$, then it was imported into ANSYS analysis software, and SWEEP mesh generation of the model was done. The material of the ring chain is defined as $25 \mathrm{MnSiNiMoAo,} \mathrm{the}$ elastic modulus is $210 \mathrm{GPa}$, the yield stress is $1.175 \mathrm{GPa}$, and the Poisson's ratio is 0.3. Applying load and boundary conditions, the equal and opposite load $10 \mathrm{~T}$ is placed on both sides of the ring chain.

After checking whether the finite element model is correct or not, the finite element model can be resolved. The analysis environment is ANSYS Workbench 14.0. Create a static structural (ANSYS), double-click the Engineering Data, modify material properties, right-click Geometry, import the model, right-click Model, , enter the Mechanical module, divide the mesh, impose load and boundary conditions, finally calculate. The display and analysis of the solver results: through ANSYS Workbench 14.0 analysis, fig. 2 and 3 are the stress contour under test load, fig. 4 and 5 are the strain contour 
under test load. From the graph we can see that the maximum stress of circular ring chain is $334.4 \mathrm{MPa}$, far less than the

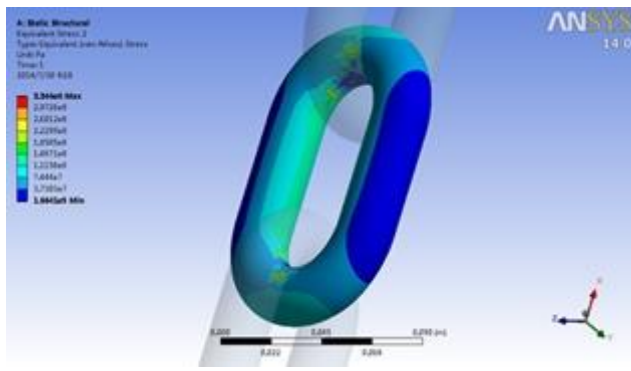

Fig. 2 stress contour under test load(1)

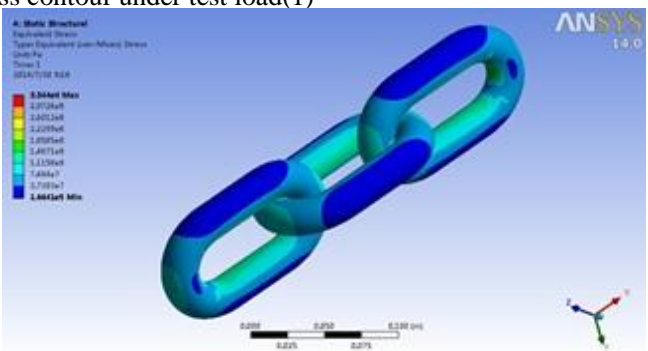

Fig. 3 stress contour under test load(1)

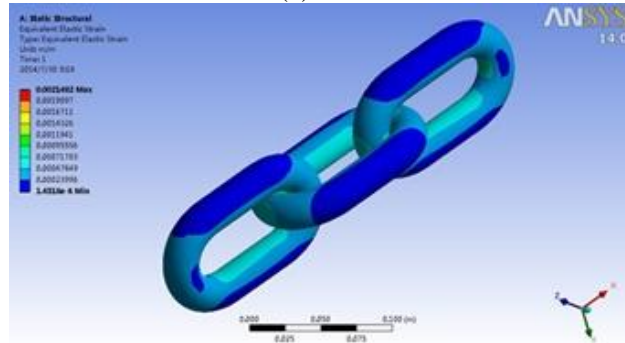

Fig. 4 strain contour under test load (1)

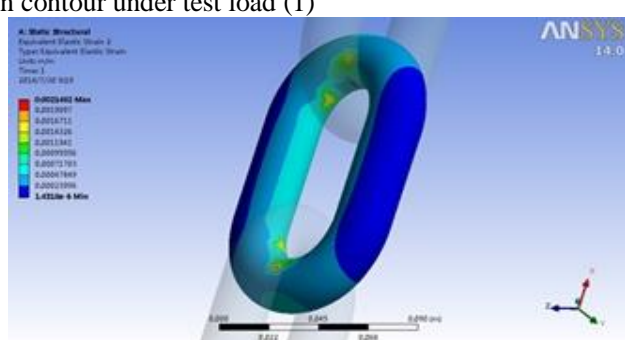

Fig. 5 strain contour under test load (2)

yield stress of the material, which meets the strength requirements, the strain is smaller by comparing with the roller chain and can better meet the high strength requirement of the high power elevator.

Through the computer optimization and simulation, the preload force and the fatigue life of different circular ring chains are obtained and also the method to improve the service life is obtained in theory. According to the stress analysis and application requirements of high power elevator, study on new materials and structure design of circular ring chain and sprocket, achieve multipoint transmission, reasonably design the traction sprocket and other mechanisms and meet the high power elevator with low speed and high load requirements. The weight balance system and the driving system of high power elevator are designed by using the modern elevator technology. Fig. 6 is the chain structure of sprocket.

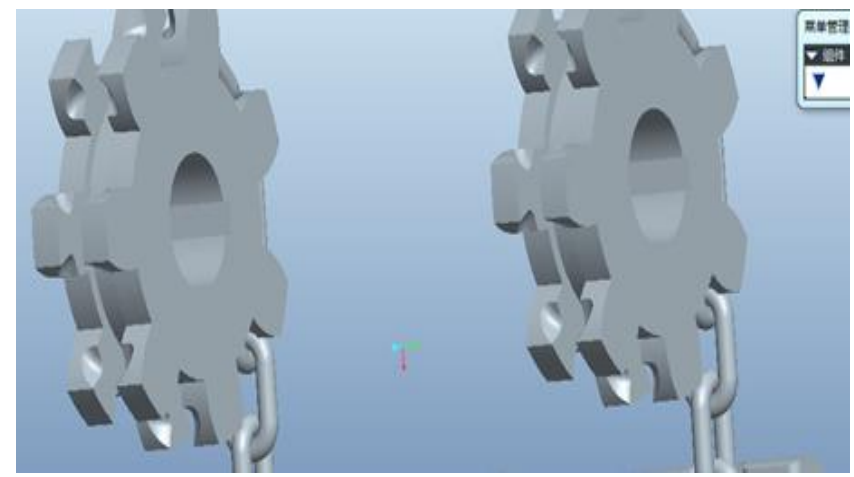

Fig. 6 chain structure of sprocket

\section{INNOVATION OF PROJECT IMPLEMENTATION}

The chain drive elevator uses the mineral high strength circular ring chain, the life normally lasts for more than 20 years, because of the bad working environment in coal mine, the chain may sustain tension, fatigue, impact, abrasion, bending stress, and also the comprehensive corrosion action from underground water, moist powdered coal, rock dust and corrosive gas exposure in the mine pit. In order to guarantee each performance index of the circular ring chain, the chain of hot-dip galvanizing for anticorrosion is used in the design. there is a mechanical drawing processing of chain after heat treatment, making the standard geometric dimensions of the chain, and this stretching can also play a role in hardening, thereby increasing the resistance to wear and corrosion.

China Zhangjiakou Coal Mining Machinery Company Limited purchased Parsons Chain Corporation's patented technology of the United Kingdom, producing large-size mining high strength ring chain c-level above with anticorrosion chain steel and achieved good results. Except for the domestic major coal mine, the chains were also exported to Australia. From 2008 adopting the technology so far, the report has not yet been received about the stress corrosion, the corrosion fatigue break of the anticorrosion chain in the coal mine. The circular ring chain uses the non-lubrication open structure in the movement, mineral high strength ring chain with higher anticorrosion requirements than elevator are used in the elevator design, the maintenance free and reliable operation under the bad environment can be realized.

Because the high power elevator will work in complex environment, temperature and the environment can not be determined in advance, so the traction rope insufficient lubrication is the main cause of premature wear of machine parts, results in the wear of traction rope and the traction pulley and even affects the normal operation of elevator. In the harsh environment the high efficiency and especially the reliable work should be first considered during the design. Through practice and investigation, open lubrication-free design theory and technique scheme were proposed for new high power elevator under the premise of sealing and lubricating the sprocket rotating parts wholly and providing with a lubricating oil filling device. By double chain driving elevator car and counter-weight, the problem of lateral force is 
solved during the operation of the elevator. Furthermore, the double chain structure and the automatic chain tension detection device can ensure the safety operation of the high power elevator in the premise of overload and single chain failure, which improve the work reliability of high power elevator system.

The circular ring chain and sprocket drive mechanism in the high power elevator are adopted to realize the maintenance free and reliable operation under the low speed and heavy load. The machine not only maintains the advantages of traditional traction rope elevator, such as energy saving, material saving, small rated torque of reducer and so on, but also overcomes the shortcomings of original traction rope elevator such as high failure rate and poor adaptability in complex environment, which has the advantages of simple structure, safety, high reliability, smooth running and good comprehensive economic effects etc. New chain drive elevator in complex environment, who has strong vitality and competitiveness, will become the ideal substitute of high power traction elevator rope elevator in complex environment and will have better market prospect and promotion value. It will also go to the domestic and foreign markets.

\section{References}

[1] Utility model patent: an elevator guide shoe for active vibration reduction. Patent No.: ZL201420311353.9, Niu Shuguang, Xue Lin, Zhuang Yu, Wang Xiaolei, Chen Jin, Wang Yongji

[2] Utility model patent: a kind of elevator guide shoe with compensation function. Patent No.: ZL2011420136420.8. Niu Shuguang, Xue Lin, Guo Lanzhong

[3] Xue Lin, Niu Shuguang; research on the cushion airbag in the elevator car, Journal of Changshu Institute Of Technology, 2014.07

[4] Wang Xiaolei, Niu Shuguang, Application of super capacitor in emergency and energy saving of elevator, Journal of Changshu Institute Of Technology, 2013.2

[5] 5.Niu Shuguang, Xue Lin, Zhou Guiyuan Study of Elevator Flexible Sheet Metal Processing Production Line Based on Open CNC System ; Applied mechanics and materals 